\title{
Cellular Interdependence
}

THE coexistence within the same animal of groups of cells with different genetic constitutions has attracted increasing attention in recent years. Immunologists, for example, recognize that the existence of mosaic individuals may help to define the conditions under which genetically different populations of cells may share the same physiological frame. Embryologists seek to know more about the circumstances under which mosaic individuals develop. The coexistence of genetically different groups of cells is then turning out to be important in the recognition of inheritable genetic defects. Now there is also the suggestion that the processes of ageing, which are known to be statistically associated with an increasing incidence of genetic abnormalities of some kinds in human reproduction, may also be linked with the increased appearance of genetic abnormalities among the body cells of living individuals. [The alternative suggestion by Dr James German, on page 516 of this issue, of the processes underlying the increasing incidence of mongolism with increasing maternal age, should not at this stage be regarded as more than what he says it is-an interesting hypothesis which deserves fuller study.] In one way and another, there has recently been a surge of interest in the coexistence of genetically different groups of cells, and it is plainly of the greatest importance to know just what factors determine the equilibrium between two such populations.

Basic biological studies by Dr D. T. Hughes of the Chester Beatty Research Institute (page 518) have demonstrated that co-operation as well as competition between cells affects the evolution of cell populations. Although interaction by cross-feeding-a situation in which cells produce substances into the culture medium which other cells take up and make use of-has previously been described in bacterial and fungal cultures, it has generally been regarded as a nuisance to be avoided, because it complicates the genetic analysis of micro-organisms. Dr Hughes, however, has shown the biological significance of interaction between cells; it is a genetically important factor in evolution, superimposed on the action of straightforward environmental selection-"survival of the fittest". The discovery of a new kind of balanced genetic polymorphism was made as a result of analysis of cell populations as a whole, carried out to determine whether there was any basis for co-operative interaction between cells. The resulting biological concept envisages a balance of cell types in which the frequencies of types of chromosomes among the cells contribute to a balance similar to that which exists in a normal cell. The situation seems to be that the balance of nature at the level of the population obeys the same laws as at the lower level of organization, within a single normal cell. Previous work on genetic polymorphism has not demonstrated this kind of balance because populations have not been considered as a whole. On the contrary, earlier work has been concerned with the frequency of characters in single cells or single organisms within a population.

It remains to be seen whether this type of polymorphism can be demonstrated in vivo in populations of organisms living in restricted environments analogous to the culture bottle--perhaps populations of mammals or plants living in geographically restricted environments such as islands would be interesting to investigate. There is, however, a possibility that fuller use could now be made of cultured cells as a model system for the study of evolution.

This new concept will also be of more immediate practical importance in clinical medicine. It should be important in the understanding of the development of mosaics and chimaeras, and in the study of tumour cell populations and their therapy-the idea of the pseudo-stemline may help to explain some of the difficulty encountered in killing off tumour cells.

\section{Geomagnetic Dynamo Energy \\ from a Geophysics Correspondent}

IT seems now generally accepted that the main geomagnetic field is produced by "dynamo" action-a magnetohydrodynamic (MHD) interaction in the Earth's liquid, conducting core which produces an essentially dipolar field whose axis coincides approximately with the rotational axis. Less firmly established is the source of the energy required to produce the fluid motions. It is fair to say that majority opinion favours thermal convection as the power source for the dynamo, although Malkus ( $J$. Geophys. Res., 68, $2871 ; 1963$ ) concluded that the driving force may well be the Earth's rotational energy operating through a differential precessional torque between mantle and core.

Stacey (Earth Plan. Sci. Letters, 3, 204; 1967) has now presented a revised estimate for the electrical resistivity of the Earth's core which suggests that Malkus's theory is the more appropriate. Any MHD theory requires the core to possess a "magnetic Reynolds number" considerably greater than unity. After making the usual numerical substitutions for core scale size, magnetic diffusivity and velocity of core motions, this means that the resistivity should be substantially smaller than $4 \times 10^{7} \mathrm{emu}$. Hitherto, estimates of core resistivity, based on extrapolation of the resistivity of iron to core temperatures and pressures, have been at least a factor of one hundred smaller than this figure, thereby satisfying the condition for an MHD interaction. Stacey points out, however, that such estimates have failed to take into account the effect of impurity elements which, if present in the core, will alloy with the iron.

A core containing ten per cent nickel alloying with 